\title{
Error Estimates for the Approximation of a Class of Variational Inequalities
}

\author{
By Richard S. Falk
}

\begin{abstract}
In this paper, we prove a general approximation theorem useful in obtaining order of convergence estimates for the approximation of the solutions of a class of variational inequalities. The theorem is then applied to obtain an "optimal" rate of convergence for the approximation of a second-order elliptic problem with convex set $K=\left\{v \in H_{0}^{1}(\Omega): v \geqslant X\right.$ a.e. in $\left.\Omega\right\}$.
\end{abstract}

1. Introduction. In this paper. we consider a method for obtaining error estimates for the approximation of a class of variational inequalities. More specifically, we let $V$ be a Hilbert space, $V^{\prime}$ its dual (with norms $\|\cdot\|_{V}$ and $\|\cdot\|_{V^{\prime}}$ respectively), and denote by $(\cdot, \cdot)$ the pairing between $V$ and $V^{\prime}$. Now, let $a(u, v)$ be a continuous, coercive, symmetric, bilinear form on $V$, i.e., there exist constants $\alpha, C_{a}>0$, such that

$$
|a(u, v)| \leqslant C_{a}\|u\|_{V}\|v\|_{V} \quad \forall u, v \in V
$$

and

$$
a(v, v) \geqslant \alpha\|v\|_{V}^{2} \quad \forall v \in V
$$

Finally, let $K$ be a closed convex subset of $V$ and $f$ an element of $V^{\prime}$. We then consider the approximation of problems of the following type:

Problem P. Find $u \in K$ such that

$$
a(u, v-u) \geqslant(f, v-u) \quad \forall v \in K .
$$

It was shown by Lions and Stampacchia in [6] that, under slightly weaker conditions (symmetry is not needed), there exists a unique solution to Problem P. Furthermore, (with symmetry) Problem $P$ is equivalent to

Problem $\mathrm{P}^{\prime}$. Find $u \in K$ such that

$$
J(u)=\inf _{v \in K} J(v) \quad \text { where } J(v)=1 / 2 a(v, v)-(f, v) .
$$

As was done in [2] by Brézis and Sibony, we shall use this second formulation

Received October 11, 1973.

AMS (MOS) subject classifications (1970). Primary 35J20, 41 A15, 41 A25, 65N30.

Copyright $\odot 1974$, American Mathematical Society 
of the problem to construct a finite-dimensional approximate problem which can be solved by mathematical programming. The original formulation will then be used to derive an error estimate for this approximation scheme. The remainder of the paper will then be devoted to showing how these results can be applied to obtain an approximation scheme and an error estimate in a specific problem.

2. Approximation Scheme and General Error Estimate. Let $V_{h}$ be a finite-dimensional subspace of $V$ with basis $\left\{w_{i}\right\}_{i=1}^{q}$. We can then write an arbitrary element $v_{h} \in V_{h}$ as $\sum_{i=1}^{q} \beta_{i} w_{i}$. Now, construct a closed convex subset $K_{h}$ of $V_{h}$ such that the following two conditions are satisfied:

(1) $K_{h}$ should reduce to a finite number of constraints on the $\beta_{i}$.

(2) $K_{h}$ should be a "good" approximation to $K$. (This condition will be made clear by the example presented in the next section.)

The approximate problem is simply

Problem $\mathrm{P}_{h}^{\prime}$. Find $u_{h} \in K_{h}$ such that

$$
J\left(u_{h}\right)=\inf _{v_{h} \in K_{h}} J\left(v_{h}\right)
$$

where $J\left(v_{h}\right)=1 / 2 a\left(v_{h}, v_{h}\right)-\left(f, v_{h}\right)$.

Note that, under condition (1), this problem becomes one of finding the minimum of a quadratic form in the $\beta_{i}$, subject to a finite number of constraints on the $\beta_{i}$, a nonlinear programming problem. In the event the constraints are linear, we have a quadratic programming problem. Note that we do not require that $K_{h}=K \cap V_{h}$, or even that $K_{h}$ be contained in $K$.

We remark that Problem $\mathrm{P}_{h}^{\prime}$ is equivalent to

Problem $\mathrm{P}_{h}$. Find $u_{h} \in K_{h}$ such that

$$
a\left(u_{h}, v_{h}-u_{h}\right) \geqslant\left(f, v_{h}-u_{h}\right) \quad \forall v_{h} \in K_{h},
$$

and that by the theorem of Lions and Stampacchia, this approximate problem also possesses a unique solution.

We now introduce a final piece of notation and then derive a general error estimate for the approximation scheme given above. Suppose that $W$ is a Hilbert space dense in $V^{\prime}$ and the injection of $W$ into $V^{\prime}$ continuous. Then there exists a continuous injection $i$ of $V$ into $W^{\prime}$ such that $i(V)$ is dense in $W^{\prime}$ and

$$
(i(v), w)_{W, w^{\prime}}=(v, w)_{V, V^{\prime}} \quad \forall v \in V, \quad \forall w \in W .
$$

We will henceforth identify $V$ with a subspace of $W^{\prime}$, dense in $W^{\prime}$ with a continuous injection map. Furthermore, we will let $(\cdot, \cdot)$ denote both the pairing between $V$ and $V^{\prime}$, and $W$ and $W^{\prime}$. With this notation, we now derive the following general error estimate. 
THeorem 1. Let $u$ and $u_{h}$ be the solutions of Problems $\mathrm{P}$ and $\mathrm{P}_{h}$, respectively. Denote by $A \in L\left(V, V^{\prime}\right)$ the map defined, for $u \in V$, by $a(u, v)=(A u, v) \forall v \in V$. Finally, suppose that $f-A u \in W$. Then,

$$
\left\|u-u_{h}\right\|_{V} \leqslant\left\{\frac{C_{a}^{2}}{\alpha^{2}}\left\|u-v_{h}\right\|_{V}^{2}+\frac{2}{\alpha}\|f-A u\|_{W}\left[\left\|u-v_{h}\right\|_{W^{\prime}}+\left\|u_{h}-v\right\|_{W^{\prime}}\right]\right\}^{1 / 2}
$$

$$
\forall v \in K \text { and } \forall v_{h} \in K_{h} .
$$

Proof. By the definitions of $u$ and $u_{h}$, we have that

$$
\begin{array}{cc}
a(u, u-v) \leqslant(f, u-v) & \forall v \in K, \\
a\left(u_{h}, u_{h}-v_{h}\right) \leqslant\left(f, u_{h}-v_{h}\right) & \forall v_{h} \in K_{h} .
\end{array}
$$

Adding these inequalities and transposing terms, we obtain

$$
a(u, u)+a\left(u_{h}, u_{h}\right) \leqslant(f, u-v)+\left(f, u_{h}-v_{h}\right)+a(u, v)+a\left(u_{h}, v_{h}\right) .
$$

Subtracting $a\left(u, u_{h}\right)+a\left(u_{h}, u\right)$ from both sides and grouping terms, we obtain

$$
\begin{aligned}
a\left(u-u_{h}, u-u_{h}\right) & \leqslant\left(f, u-v_{h}\right)+\left(f, u_{h}-v\right)-a\left(u, u_{h}-v\right)-a\left(u_{h}, u-v_{h}\right) \\
& =\left(f, u-v_{h}\right)+\left(f, u_{h}-v\right)-a\left(u, u_{h}-v\right)-a\left(u, u-v_{h}\right)+a\left(u-u_{h}, u-v_{h}\right) \\
& =\left(f-A u, u-v_{h}\right)+\left(f-A u, u_{h}-v\right)+a\left(u-u_{h}, u-v_{h}\right)
\end{aligned}
$$

by the definition of $A$ and the fact that $K$ and $K_{h} \subset V$. Since by assumption $f-A u \in W$, we have, using the continuity and coercivity of the bilinear form $a(u, v)$, that

$$
\begin{aligned}
\alpha\left\|_{u}-u_{h}\right\|_{V}^{2} \leqslant & \|f-A u\|_{W}\left\|u-v_{h}\right\|_{W^{\prime}}+\|f-A u\|_{W}\left\|_{h}-v\right\|_{W^{\prime}} \\
& +C_{a}\left\|u-u_{h}\right\|_{V}\left\|_{u}-v_{h}\right\|_{V} .
\end{aligned}
$$

Since

$$
C_{a}\left\|u-u_{h}\right\|_{V}\left\|u-v_{h}\right\| \leqslant \frac{\alpha}{2}\left\|u-u_{h}\right\|_{V}^{2}+\frac{C_{a}^{2}}{2 \alpha}\left\|u-v_{h}\right\|_{V}^{2}
$$

we have that

$$
\begin{array}{r}
\frac{\alpha}{2}\left\|u-u_{h}\right\|_{V}^{2} \leqslant\|f-A u\|_{W}\left\|u-v_{h}\right\|_{W^{\prime}}+\|f-A u\|_{W}\left\|u_{h}-v\right\|_{W^{\prime}}+\frac{C_{a}^{2}}{2 \alpha}\left\|u-v_{h}\right\|_{V}^{2} \\
\forall v \in K \quad \text { and } \forall v_{h} \in K_{h} .
\end{array}
$$

The theorem follows immediately.

3. An Application. We begin with some notation. Let $\Omega$ be a bounded convex domain in $R^{2}$ with $C^{2}$ boundary $\partial \Omega$. Let $m$ be a nonnegative integer and let 
$C^{\infty}(\bar{\Omega})$ denote the set of infinitely differentiable functions on $\bar{\Omega}$. Then $H^{m}(\Omega)$ will denote the completion of $C^{\infty}(\bar{\Omega})$ in the norm

$$
\|\phi\|_{m, \Omega}=\left(\sum_{|\alpha| \leqslant m}\left\|D^{\alpha} \phi\right\|_{0, \Omega}^{2}\right)^{1 / 2}
$$

where $\|\phi\|_{0, \Omega}=\left(\int_{\Omega}|\phi|^{2} d x\right)^{1 / 2}$.

Now, let $C_{0}^{\infty}(\Omega)$ be the set of infinitely differentiable functions with compact support on $\Omega$ and denote the completion of $C_{0}^{\infty}(\Omega)$ in the above norm by $H_{0}^{m}(\Omega)$.

For $m$ a negative integer, we define $H^{m}(\Omega)$ as the completion of $C^{\infty}(\bar{\Omega})$ with respect to the norm

$$
\|\phi\|_{m, \Omega}=\sup _{\chi \in C_{0}^{\infty}(\Omega)} \frac{(\phi, \chi)}{\|\chi\|_{-m, \Omega}}
$$

where $(\phi, \chi)=\int_{\Omega} \phi \chi d x$.

For $m$ a negative integer, we note that $H^{m}(\Omega)=\left[H_{0}^{-m}(\Omega)\right]^{\prime}$, the dual space of $H_{0}^{-m}(\Omega)$.

We now consider the special case when Problem $P$ is defined in the following setting. Let $V=H_{0}^{1}(\Omega), V^{\prime}=H^{-1}(\Omega)$, and $W=W^{\prime}=L^{2}(\Omega)$. For $u, v \in H_{0}^{1}(\Omega)$, let

$$
a(u, v)=\sum_{i, j=1}^{2} \int_{\Omega} a_{i j}(x) u_{x_{i}} v_{x_{j}} d x+\int_{\Omega} c(x) u v d x
$$

where

$$
\sum_{i, j=1}^{2} a_{i j} \xi_{i} \xi_{j} \geqslant \bar{\alpha}|\xi|^{2} \quad \forall \xi \in R^{2}
$$

and some $\bar{\alpha}>0$. Suppose that $a_{i j}=a_{j i}$ so that the bilinear form $a(u, v)$ is symmetric, and that $a_{i j} \in C^{1}(\bar{\Omega})$ and $c \in L^{\infty}(\Omega)$. Then, there exists a constant $C_{a}>0$ such that

$$
|a(u, v)| \leqslant C_{a}\|u\|_{1, \Omega}\|v\|_{1, \Omega} \quad \forall u, v \in H_{0}^{1}(\Omega)
$$

Further, suppose that $c(x) \geqslant \lambda$ where $\lambda$ is either nonnegative or negative with a sufficiently small absolute value so that $a(u, v)$ is coercive on $H_{0}^{1}(\Omega)$, i.e.,

$$
a(v, v) \geqslant \alpha\left\|_{v}\right\|_{1}^{2} \quad \forall v \in H_{0}^{1}(\Omega)
$$

and some constant $\alpha>0$.

Let $A$ be the operator defined by $A u=-\Sigma_{i, j=1}^{2}\left(a_{i j} u_{x_{i}}\right)_{x_{j}}+c u$. Then, for $u, v \in H_{0}^{1}(\Omega), a(u, v)=(A u, v)$, and since $a_{i j}=a_{j i}, A$ is a formally selfadjoint secondorder elliptic operator mapping $H_{0}^{1}(\Omega) \rightarrow H^{-1}(\Omega)$.

Finally, suppose that $f \in L^{2}(\Omega)$ and $\chi$ is a given function in $H^{2}(\Omega)$ such that $\chi \leqslant 0$ on $\partial \Omega$. In terms of the above notation, we will consider the approxima- 
tion of Problem P where

$$
K=\left\{v \in H_{0}^{1}(\Omega): v \geqslant \chi \text { a.e. in } \Omega\right\} .
$$

In the process of developing an approximation scheme and error estimate for this problem, we will need to make use of a regularity result for the solution $u$. Brézis and Stampacchia have shown in [3] that under the conditions which we have imposed, the solution $u$ of Problem P belongs to $H^{2}(\Omega)$. Furthermore, we have the estimate

$$
\|A u\|_{L^{2}(\Omega)} \leqslant \max \left\{\|f\|_{L^{2}(\Omega)}, \sigma\right\}
$$

where $\sigma$ is the solution of the equation

$$
\sigma=\|\sup (A \chi, 0)\|_{L^{2}(\Omega)}\left(\sigma+\|f\|_{L^{2}(\Omega)}\right) /\left(\sigma-\|f\|_{L^{2}(\Omega)}\right) .
$$

We remark that since $\|u\|_{2, \Omega} \leqslant C\|A u\|_{L^{2}(\Omega)}$ for some constant $C$, (4) also yields an estimate for $\|u\|_{2, \Omega}$ which depends only on the data of the problem.

Since it will have a bearing on the optimality of our final error estimate, we also remark that Lewy and Stampacchia have given a simple counterexample in [5] to show that, in general, the solution $u$ of Problem $\mathrm{P}$ will not belong to $C^{2}(\Omega)$, even under additional regularity assumptions on the data of the problem.

The approximation scheme we use for this problem will be of the general type described in Section 2, i.e., we will replace Problem P by a minimization problem of type $\mathrm{P}_{h}^{\prime}$ which can be solved by mathematical programming techniques. To completely define our approximation scheme for the case we are considering here, we need only give explicit choices for the finite-dimensional subspace $V_{h} \subset H_{0}^{1}(\Omega)$ and the approximate convex set $K_{h} \subset V_{h}$. We first describe the construction of $V_{h}$.

Let $h, 0<h<1$, be a parameter, and, for each value of $h$, let $\Omega_{h}$ be a polygon inscribed in $\Omega$ with all its vertices lying on $\partial \Omega$ and each side of the polygon of length less than or equal to $h$. Now subdivide $\Omega_{h}$ into triangles $T_{q}, q=1, \cdots$, $N_{h}$, such that the following two conditions are satisfied:

The ratio of any two triangle sides in the triangulation is bounded by a constant $b$ independent of $h$.

All the angles in the triangulation are greater or equal to some angle $\phi$ independent of $h$.

Using the terminology of Nitsche [9], we will henceforth refer to a triangulation satisfying conditions (5) and (6) as $\phi-b$ regular.

Now, define $V_{h}=\left\{v_{h}: v_{h}\right.$ is linear in each triangle $T_{q}, q=1, \cdots, N_{h}$, continuous on $\Omega$, and $v_{h}=0$ in $\Omega-\Omega_{h}$. Clearly, $V_{h}$ is a finite-dimensional subspace of $H_{0}^{1}(\Omega)$. Finally, define $K_{h}=\left\{v_{h} \in V_{h}: v_{h} \geqslant \chi\right.$ at every vertex of each triangle $T_{q}, q=1, \cdots, N_{h}$. Clearly, $K_{h}$ is a closed convex subset of $V_{h}$ and 
satisfies condition (1) of Section 2. Since $K_{h}$ reduces to a finite number of linear constraints, the approximate minimization problem $\mathrm{P}_{h}^{\prime}$ becomes a problem in quadratic programming.

The remainder of this section will be devoted to proving the following error estimate for the approximation scheme just presented.

THeOREM 2. Let $u$ and $u_{h}$ be the respective solutions of Problems $\mathbf{P}$ and $\mathbf{P}_{h}$ with $K$ and $K_{h}$ defined as above. Then there exists a constant $C$ independent of $u$ and depending on the data $\Omega, f, \chi, a_{i j}$, and $c$ such that

$$
\left\|u-u_{h}\right\|_{1, \Omega} \leqslant C h \text {. }
$$

To prove this theorem, we will need to make use of the following results which can be found in Nitsche [9].

Lemma 1. Let $W=W\left(r_{1}, r_{2}\right)\left(0<r_{1}<r_{2}<\infty\right)$ be the class of convex domains $\Omega \subset R^{2}$ with piecewise smooth boundary that satisfy $O_{1} \subseteq \Omega \subseteq O_{2}$ where $O_{1}, O_{2}$ are two concentric circles of radii $r_{1}$ and $r_{2}$, respectively. Then, if $\Omega$ and $\Omega^{\prime} \in$ $\omega$ with $\operatorname{dist}\left(\partial \Omega, \partial \Omega^{\prime}\right) \leqslant \delta$ and $u \in H_{0}^{1}(\Omega) \cap H^{2}(\Omega)$, we have

$$
\|u\|_{k, \Omega-\Omega^{\prime}} \leqslant C \sqrt{\delta}^{2-k}\|u\|_{2, \Omega}
$$

for $k=0,1$ and $C$ depending only on $r_{1}$ and $r_{2}$.

LEMмA 2. Let $T$ be a polygon and $T_{h} a \phi-b$ regular triangularization of $T$ where $h$ denotes the maximum of the sides of all the triangles making up $T_{h}$. If $u \in H^{2}(T)$, then

$$
\left\|u-S_{h}^{u}\right\|_{k, T} \leqslant C(\phi, b) h^{2-k}\|u\|_{2, T}
$$

where $S_{h}^{u}$ is the piecewise linear interpolant of $u$, i.e., $S_{h}^{u}=u$ at the vertices of the triangulation, and $S_{h}^{u}$ is linear in each triangle.

The proof of Theorem 2 will follow from an application of the general error estimate given in Theorem 1. In the setting we are in, (3) becomes

$$
\begin{array}{r}
\left\|u-u_{h}\right\|_{1, \Omega} \\
(10) \leqslant\left\{\frac{C_{a}^{2}}{\alpha^{2}}\left\|u-v_{h}\right\|_{1, \Omega}^{2}+\frac{2}{\alpha}\|f-A u\|_{L^{2}(\Omega)}\left[\left\|u-v_{h}\right\|_{L^{2}(\Omega)}+\left\|u_{h}-v\right\|_{L^{2}(\Omega)}\right]\right\}^{1 / 2} \\
\forall v \in K, \quad \forall v_{h} \in K_{h} .
\end{array}
$$

To apply this result, we will need estimates for the quantities $\left\|u-v_{h}\right\|_{1, \Omega}$, $\left\|u-v_{h}\right\|_{L^{2}(\Omega)}$, and $\left\|u_{h}-v\right\|_{L^{2}(\Omega)}$, for some $v \in K$ and some $v_{h} \in K_{h}$.

In the lemmas which follow, $C$ will denote a generic constant, not necessarily the same in any two places.

Leмма 3. Let $v_{h}$ be the unique element in $V_{h}$ such that $u=v_{h}$ at all 
vertices of each triangle $T_{q}, q=1, \cdots, N_{h}$. Then $v_{h} \in K_{h}$ and

$$
\begin{gathered}
\left\|u-v_{h}\right\|_{L^{2}(\Omega)} \leqslant C h^{2}\|u\|_{2, \Omega}, \\
\left\|u-v_{h}\right\|_{1, \Omega} \leqslant C h\|u\|_{2, \Omega},
\end{gathered}
$$

where $C$ is a constant independent of $h$ and $u$.

Proof. By the regularity result for $u$ and the Sobolev imbedding theorem, both $u$ and $\chi$ are continuous. Since $u \in K, u(x) \geqslant \chi(x) \forall x \in \bar{\Omega}$. Hence, by the definition of $v_{h}$, we know that, if $\omega$ is a vertex of one of the triangles $T_{q}, v_{h}(\omega)=$ $u(\omega) \geqslant \chi(\omega)$, which implies that $v_{h} \in K_{h}$. To obtain (11) and (12), we set for $k=0,1$

$$
\left\|u-v_{h}\right\|_{k, \Omega}^{2}=\left\|u-v_{h}\right\|_{k, \Omega_{h}}^{2}+\left\|u-v_{h}\right\|_{k, \Omega-\Omega_{h}}^{2}
$$

and apply Lemmas 1 and 2 .

From Lemma 2, $\left\|u-v_{h}\right\|_{k, \Omega_{h}} \leqslant C(\phi, b) h^{2-k}\|u\|_{2, \Omega_{h}}$. Using Lemma 1 and the fact that $v_{h}=0$ on $\Omega-\Omega_{h}$, we have

$$
\left\|u-v_{h}\right\|_{k, \Omega-\Omega_{h}}=\|u\|_{k, \Omega-\Omega_{h}} \leqslant C \sqrt{\delta}^{2-k}\left\|_{u}\right\|_{2, \Omega} \quad \text { where } \delta=\operatorname{dist}\left(\partial \Omega, \partial \Omega_{h}\right)
$$

and $C$ is independent of $u$ and $\delta$. Furthermore, since $\partial \Omega \in C^{2}$ and the largest side of the inscribed polygon $\Omega_{h}$ is $\leqslant h, \delta \leqslant C(\partial \Omega) h^{2}$ where $C(\partial \Omega)$ is a constant depending only on $\partial \Omega$. Hence, we have

$$
\left\|u-v_{h}\right\|_{k, \Omega}^{2} \leqslant\left[C(\phi, b) h^{2-k}\|u\|_{2, \Omega_{h}}\right]^{2}+\left[C(\partial \Omega) h^{2-k}\|u\|_{2, \Omega}\right]^{2}
$$

Since $\|u\|_{2, \Omega_{h}} \leqslant\|u\|_{2, \Omega}$, we have finally that $\left\|u-v_{h}\right\|_{k, \Omega} \leqslant C h^{2-k}\|u\|_{2, \Omega}, k=$ 0,1 , for some constant $C$ independent of $h$ and $u$.

We now turn our attention to deriving an estimate for $\left\|u_{h}-v\right\|, v \in K$, where $u_{h}$ is the solution of the approximate problem $\mathrm{P}_{h}^{\prime}$.

Lemm A 4. Let $u_{h}$ be the solution of Problem $\mathbf{P}_{h}^{\prime}$ and let $v=\sup \left\{u_{h}, \chi\right\}$.

Then $v \in K$ and

$$
\begin{aligned}
& \left\|u_{h}-v\right\|_{L^{2}(\Omega)} \leqslant C h^{2} \text { for some constant } C \\
& \text { independent of } u_{h} \text { and } h .
\end{aligned}
$$

Proof. Since $u_{h} \in H_{0}^{1}(\Omega)$ and $\chi \in H^{2}(\Omega)$ with $\chi \leqslant 0$ on $\partial \Omega$, clearly, $v \in K$.

Now

$$
\begin{aligned}
\left\|u_{h}-v\right\|_{L^{2}(\Omega)}^{2} & =\left\|u_{h}-v\right\|_{L^{2}\left(\Omega_{h}\right)}^{2}+\left\|u_{h}-v\right\|_{L^{2}\left(\Omega-\Omega_{h}\right)}^{2} \\
& =\left\|u_{h}-v\right\|_{L^{2}\left(\Omega_{h}\right)}^{2}+\|\sup (0, \chi)\|_{L^{2}\left(\Omega-\Omega_{h}\right)}^{2},
\end{aligned}
$$

since $u_{h}=0$ in $\Omega-\Omega_{h}$. Now 


$$
\begin{aligned}
u_{h}-v & =0 \quad \text { when } u_{h} \geqslant \chi, \\
& =u_{h}-\chi \quad \text { when } u_{h}<\chi .
\end{aligned}
$$

Let $S_{h}^{\mathrm{X}}$ be the unique element in $V_{h}$ such that $S_{h}^{\mathrm{X}}$ interpolates $\chi$ at the vertices of each triangle $T_{q}, q=1, \cdots, N(h)$. Since $u_{h} \in K_{h}, u_{h} \geqslant \chi=S_{h}^{\chi}$ at these vertices. But since $S_{h}^{\chi}$ and $u_{h}$ are piecewise linear, $u_{h} \geqslant S_{h}^{\chi} \forall x \in \Omega_{h}$. Hence, on $\Omega_{h}$, when $u_{h}-\chi<0,0>u_{h}-v=u_{h}-\chi=u_{h}-S_{h}^{\chi}+S_{h}^{\chi}-\chi \geqslant S_{h}^{\chi}-\chi$ which implies that, $\forall x \in \Omega_{h},\left|u_{h}-v\right| \leqslant\left|\chi-S_{h}^{\chi}\right|$ and hence that $\left\|u_{h}-v\right\|_{L^{2}\left(\Omega_{h}\right)}^{2} \leqslant\left\|\chi-S_{h}^{\chi}\right\|_{L^{2}\left(\Omega_{h}\right)}^{2}$.
Thus we have

$$
\left\|u_{h}-v\right\|_{L^{2}(\Omega)}^{2} \leqslant\left\|\chi-S_{h}^{\chi}\right\|_{L^{2}\left(\Omega_{h}\right)}^{2}+\| \sup (0, \chi)_{L^{2}\left(\Omega-\Omega_{h}\right)}^{2} .
$$

But by Lemma 2,

$$
\left\|\chi-S_{h}^{\chi}\right\|_{L^{2}\left(\Omega_{h}\right)} \leqslant C h^{2}\left\|_{\chi}\right\|_{2, \Omega_{h}} \leqslant C h^{2}\left\|_{\chi}\right\|_{2, \Omega}
$$

and by a slight modification of Lemma 1 , one can show

$$
\|\sup (0, \chi)\|_{L^{2}\left(\Omega-\Omega_{h}\right)} \leqslant C h^{2}\left\|_{\chi}\right\|_{2, \Omega} \text {. }
$$

Hence $\left\|u_{h}-v\right\|_{L^{2}(\Omega)} \leqslant C h^{2}\left\|_{\chi}\right\|_{2, \Omega} \leqslant C h^{2}$ where $C$ is independent of $u_{h}$ and $h$. We now note that Theorem 2 follows immediately from Lemmas 3 and 4 and the a priori estimates for $u$ and $A u$ stated previously.

4. Conclusions. We remark that since the best approximation to elements in $H^{2}(\Omega)$ by elements in $V_{h}$ is of order $h$ in the $H^{1}(\Omega)$ norm, our estimate is optimal in the sense that it duplicates up to a multiplicative constant the best approximation properties of the subspace. We further note that by the counterexample of Lewy and Stampacchia, no higher-order accuracy can be achieved by using better subspaces, e.g., higher-order splines, since this would require additional regularity of the solution.

Acknowledgement. This work is based in part on the author's Ph.D. thesis. The author would like to express his appreciation to his thesis advisor, Professor J. H. Bramble, for his help and encouragement during the course of that work.

Department of Mathematics

Rutgers University

New Brunswick, New Jersey 08903

1. J. P. AUBIN, “Approximation of variational inequalities," Functional Analysis and Optimization (edited by E. R. Caianiello), Academic Press, New York, 1966, pp. 7-14. MR 35 \#4743.

2. H. BREZIS \& M. SIBONY, "Equivalence de deux inéquations variationnelles et applications," Arch. Rational Mech. Anal., v. 41, 1971, pp. 254-265.

3. H. BRÉZIS \& G. STAMPACCHIA, "Sur la regularité de la solution d'inéquations elliptiques," Bull. Soc. Math. France, v. 96, 1968, pp. 153-180. MR 39 \#659. 
4. R. S. FALK, Approximate Solutions of Some Variational Inequalities with Order of Convergence Estimates, Ph. D. Thesis, Cornell University, Ithaca, N. Y., 1971.

5. H. LEWY \& G. STAMPACCHIA, "On the regularity of the solution of a variational inequality," Comm. Pure Appl. Math., v. 22, 1969, pp. 153-188. MR 40 \#816.

6. J. L. LIONS \& G. STAMPACCHIA, "Variational inequalities," Comm. Pure Appl. Math., v. 20, 1967, pp. 493-519. MR 35 \#178.

7. U. MOSCO, “Approximation of the solutions of some variational inequalities," Ann. Scuola Norm. Sup. Pisa, (3) v. 21, 1967, pp. 373-394; erratum, ibid. (3) v. 21, 1967, p. 765. MR 37 \#1966.

8. U. MOSCO, "Convergence of convex sets and of solutions of variational inequalities," Advances in Math., v. 3, 1969, pp. 510-585. MR 45 \#7560.

9. J. NITSCHE, "Lineare Spline-Funktionen und die Methoden von Ritz fur elliptische Randwertprobleme," Arch. Rational Mech. Anal., v. 36, 1970, 348-355. MR 40 \#8250. 